\title{
The research on personalized tourism demand model based on user roles
}

\author{
Wenlong Feng, Yucong Duan, Menxing Huang \\ College of information science and Technology \\ HaiNan University \\ Haikou,.China \\ fwlfwl@163.com
}

\begin{abstract}
According to the characteristic that individual user data is less in tourism service, a personalized demand modeling mechanism is proposed based on user roles. The mechanism researches the existing historical data, defines demand hierarchy and category and establishes the requirement set. It also analyzes relationship between user roles and requirements and gets the mapping relation between roles and requirements. The feedback mechanism is used for real-time dynamic adjustment demand set and mapping relationship according to the change of data. Simulation results show that the mechanism can realize the combination of user roles and requirements, and improve the accuracy of user's personalized needs.
\end{abstract}

Keywords—user; role; personalized needs; modeling; tourism

\section{INTRODUCTION}

With the development of enterprise informatization and modern service industry, the traditional mode of production and service concept in the production-centered is not more and more adapt to the fierce market competition. Providing personalized service differ in thousands of ways is key to enterprises that can obtain the user and be successful [1]. The premise to realize the personalized service is to obtain personalized needs accurate. There are two ways to obtain user demand: one is the dominant access that can do through user interview, feedback etc.. Another is the recessive access that can do in the rules of logic deduction according to the user's history, browsing behavior or personality [2]. The actual implementation of dominant access is very difficult that needs a large number of users to actively participate in. Recessive access does not require user direct involvement, has high intelligent, become the main way of personalized demand acquisition. The differences in the difficulty level of implicit acquisition modes for different industries get big. For example of library service, due to the resource has been classified, catalogued, borrowing behavior is often regular behavior, the historical data is abundant, the records are also easy to get, so the recessive access is easy to implement [4]. But for tourism services, as most users tend to be a one-time, and data is little, so there is certain difference between user real demand and the recessive demand by system according to historical data.
This paper proposes a mechanism to obtain personalized travel needs of users based on roles. The mechanism fully considers the characteristics of tourism industry, is based on a large amount of historical data, combines with the feature of user role, guarantees the consistency of demand and role, and improves the accuracy of the personalized needs

\section{PERSONALIZED TRAVEL SERVICE DEMAND}

\section{A. Demand characteristics}

Tourism information system has two characteristics: one is that the whole historical data and scale of user are large. The number of users of some tourism information system has reached hundreds of thousands of [5]. Two is that a single user's history is less in quantity. The statistics show that the registered user travels once every two years on average at present. Therefore, the traditional mode that single user demand is obtained by the logical rules according single users historical data is not adapt to travel services, especially for those new user. Design of user travel information system uses multi role structure and users will provide role information at the time of registration. Despite the large number of users, but the number of role types is limited, so there is a certain relationship between the role types and historical demand data. Therefore, it can reduce mapped relationship between historical demand data and the role types, and obtain user's recessive personalized needs according to the user role. Bigger system data is, the personalized demand obtained by this way is closer to the real user demand. This approach can also push the personalized needs for the new user.

\section{B. The demand structure}

Tourist Personalized needs includes two levels: one is the overall needs, consisting of tourist routes needs, such as Hainan three tour, weekend tour in Sanya etc. The two is the entity specific needs, including food, shelter, entertainment, shopping, and play etc. The tourism line needs consists of multiple entities needs, such as hotels, attractions needs. The tourism line needs is based on the entity needs, and can be obtained through the entity needs configuration [6]. Tourism 
information system can be based on user role information to obtain the overall needs or entity needs. Needs structure as shown below:

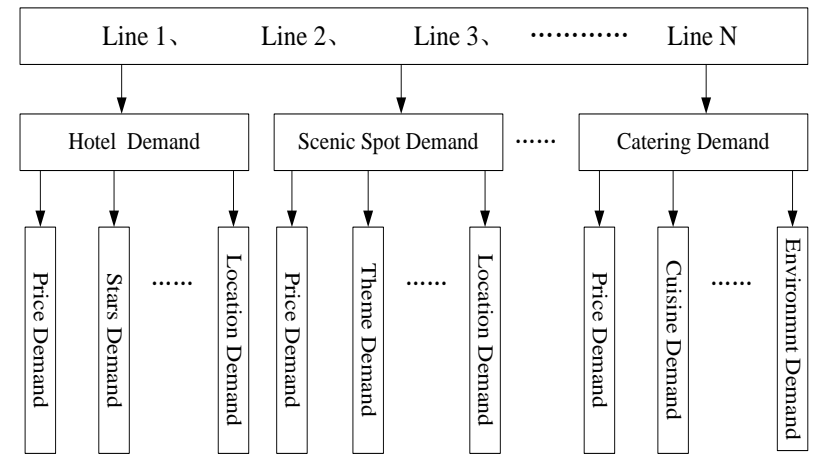

Figure 1: The structure of demand

\section{PERSONALIZED DEMAND MODELING}

The personalized travel demand model based on user roles consists of two modules: demand set module and mapping module. The demand set module is to analyze, treat and classify the large historical demand information, and obtain demand category that meets the specification requirement. The demand set includes two kinds of demand: tourism line demand and entity demand. Mapping module is to find out the relationship between the role and the demand set, link role to demand category, and indirectly obtain the user implicit demand.

\section{A. Establishment the demand set}

Define abbreviations and acronyms the first time they are used in the text, even after they have been defined in the abstract. Abbreviations such as IEEE, SI, MKS, CGS, sc, dc, and rms do not have to be defined. Do not use abbreviations in the title or heads unless they are unavoidable.

1. The entity demand modeling

The demand is expressed by the form of a key-value: a entity demand has $n$ parameters and the value of each parameter is known, then the demand can be expressed as:

$$
A\left[\left(p_{1}, v_{1}\right)\left(p_{2}, v_{2}\right),\left(p_{3}, v_{3}\right) \ldots \ldots\left(p_{n}, v_{n}\right)\right]
$$

$\mathrm{P}$ is the parameter name, $\mathrm{V}$ is value of parameter. The parameter values can be numeric, character and Boolean. For example, the hotel price is numeric, hotel environment is the character, the hotel facility (if equipped with swimming pool) is the Boolean type. It is easy to handle numerical parameter and Boolean parameter. Character parameter is description that is processed by system and follows certain specification.

The number of entity in the system demand is very great, so the workload for processing, classified these demand is very heavy. The classification granularity is the main consideration factor for demand classification. Size is too large, the classification of the quantity is too small, and it can not fully reflect the characteristics of the individualized demand. Size is too small, the complex degree of system processing is high, it is difficult to realize. This system puts forward a classification method of the entity demand that have two level granularities, the algorithm is as follows:

(1) The system is initialized that constructs a demand set marked $\mathrm{S}$ that its record is zero, and then it counts the number of all demand records marked $n$ in system.

(2) Parameter types and values of $n$ demand records are counted. If there exists $m$ records that parameter types and number of every record are same,, the $m$ records initially is consider as the same type of demand. This is the first layer of classification granularity.

(3) It continues to classify the m records. If there exists w records that parameter order of every record is same, the $\mathrm{w}$ records is determined to be same type of demand. This is the second layer of classification granularity.

(4) After processed n records, the number of demand type is $\mathrm{z}$, and add all demand types into $\mathrm{S}$.

Using two layer granularity classifications can improve the fit between the demand and the real user needs. For example, when three users such as A, B, C are booking Hotel, the parameters that are concerned by them are same, and are the hotel price, environment and geographical position. Although the number and types of parameters are same, there are differences in demand because of different the economic capacity, interests and preferences of the three users. User A may consider the price factor first, followed by the location and environment. User B will give priority to environmental factors, followed by the environment and geographical position. The parameters order that user $\mathrm{C}$ pays attention to is geographic location, price and environment.

\section{The line demand modeling}

The line demand is high-level demand, is based on the entity demand. The line demand modeling also includes history record demand and classification processing. The entity demand modeling generally refers to 6 kinds of entities. Although procedure of line demand modeling is similar to the entity demand, it is more complicated due to line number, large time span and configuration on the entity demand. The line demand modeling uses classification method of three layers granularity, the first layer is the line number, the second layer is the number and type of entity, the third layer is the entity ranking. The algorithm is as follows:

(1) The system is initialized that constructs a demand set marked S that its record is zero, then it counts the number of all line demand records and classifies all records according to first layer granularity in system.

(2) It continues to do second layer granularity classification and counts the number of demand records for each line. If there exists $m$ records that each of $m$ records has the same quantity and type of entity and each entity has a 
definite value, the $\mathrm{m}$ records initially is considered as the same type of line demand.

(3) The third layer granularity classification: continuing to classify $M$ records, if there exists $w$ records that the entity order of each record are same, then the $\mathrm{w}$ records is determined as the same type of line demand.

(4) After processed all the line records, the number of line demand type is marked $\mathrm{z}$, and add it into line demand set.

The demand modeling is shown in figure 2:

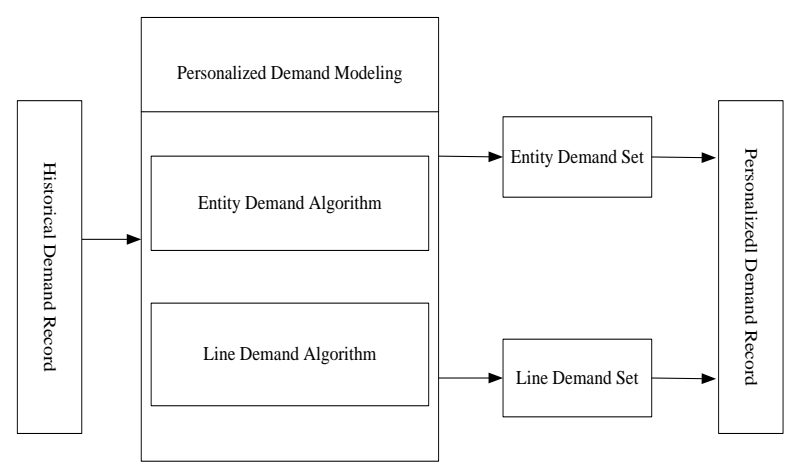

Figure 2 Demand modeling

\section{B. Establishing mapping rules}

A single user's historical demand record is small, and it is difficult to derive the user personalized needs from historical demand record. It can obtain user personalized demand indirectly by mapping rules that reflects relation between user and role. The user data structure designed in this paper is divided into two parts: basic information and character information. The basic information includes name, phone, zip code, it doesn't matter user personalized information. The role information including occupation, income, preferences, will have an impact on the user's personalized needs. Therefore, the design of role is the key research contents. The role uses the role group structure, namely divides roles into different groups according to the personal information of users [7]. The role group may be a group as an occupational category group, including civil servants, teachers, staff and other roles. It can also classify role group according to the income such as high income groups, white-collar income groups and blue-collar income groups.

\section{The single role mapping}

Role of registered users is single, belongs to a group of users. Because the number of user is large and history data is big in system, demands of each role have a certain scale. The single role mapping is to process these demands, to find the demand set that is most suitable for the role, and sort according to the degree of correlation. The algorithm consists of two steps: the first step is mapping algorithm, the second step is the recommendation algorithm. The mapping algorithm is as follows:

(1) Quantity demanded for role A is marked $\mathrm{N}_{\mathrm{A}}$, and are classified. Classification method is to use demand modeling methods, mainly consider the number, the types and the order of parameters.

(2) $\mathrm{N}$ demands are divided into a, b, c..., and the number of users for each demand are $\mathrm{N}_{\mathrm{a}}, \mathrm{N}_{\mathrm{b}}, \mathrm{N}_{\mathrm{c} . . ., \text {, so }}$ It can be expressed as:

$$
N_{A}\left[N_{a}, N_{b}, N_{c} \ldots \ldots\right]
$$

(3) Counting quantity of each demand respectively, and calculating the probabilities, as shown below:

$$
p_{a}=\frac{M_{a}}{N_{A}} \quad p_{b}=\frac{M_{b}}{N_{A}} \quad p_{c}=\frac{M_{c}}{N_{A}}
$$

Sorting these probabilities according to their numeric value, if

$$
p_{a}>p_{b}>p_{c}>\ldots \ldots
$$

Then it can draw the mapping relationship between type A and demand set:

$$
A \rightarrow N_{A}\left(p_{a}, p_{b}, p_{c}, \ldots . .\right)
$$

Mapping algorithm establishes the relationship between user roles and demand categories, and categories can be sorted by probability. But for users, in addition to the number, the type and the order of parameters, their personalized demand is also reflected in the parameters values. For example, when two users are booking hotel, parameters they concerns are completely consistent, and order of parameters is price, location, and environment. But the price range still exist differences. Therefore, it needs further treatment on the mapping demand set that sorts each kind of demand according to demand size, and recommends the most appropriate individual needs to user. Recommendation algorithm is as follows:

(1) The number of category a under role $A$ is $N_{a}$, and parameter types are $T_{1}, T_{2} . . T_{m}$.

(2) if $T_{1}$ is a numeric parameter, then the following processing is:

$$
\overline{T_{1}}=\frac{\sum_{i=1}^{N_{a}} T_{1 i}}{N_{a}}
$$

$\mathrm{T} 1 \mathrm{i}$ is specific value of parameters in $\mathrm{T} 1$

(3) Selecting parameter values and setting experience coefficient as E. If

$$
\left|T_{1 i}-\overline{T_{1}}\right|>E
$$


Then deleting the parameters and repeating steps (2) and (3), until all the values of the parameters selection is complete.

(4) Sorting parameter values according to the distance from $\bar{T}_{1}$. If a parameter is closer to $\bar{T}_{1}$, it's order is more top. It can be expressed as:

$$
T_{1}\left(T_{11}, T_{12}, T_{13}, \ldots . . T_{1 W}\right)
$$

Processing of Boolean and character parameter of system is the same that Boolean parameter is a special case of character parameter. Processing of character parameter is different from numerical parameter, and it uses the mathematical statistics method. First of all, coding the values of the each parameter, counting probability of each code, and then sorting these codes according to probability.

\section{Multiple roles mapping}

The system allows user to have multiple roles, and the personalized demand is obtained through multi roles mapping. Multi roles mapping is based on single role mapping, and carries out comprehensive treatment of all demand category under multiple roles. The algorithm is as follows: first, the system counts the number of demand for multiple roles marked N. Second, the system processes $\mathrm{N}$ needs in a single role mapping processing.

Role mapping is actually carried out through two steps: mapping algorithm carries out selection of type, order and the number of demand parameter, determines demand categories, and sorts the demand categories. Recommendation algorithm realizes selection of parameter value for a demand category, and sorts parameter values. Through these two steps, system can obtain the personalized needs of users around rolecantered. Role mapping is shown as below:

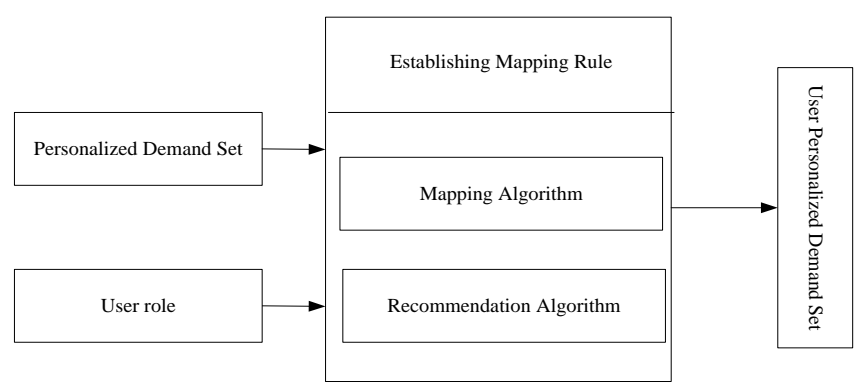

Figure 3 Establishing mapping rules

\section{CONCLUSIONS}

The modeling mechanism based user's role for user personalized demand adapts to the characteristics of tourism industry, and overcomes the defect of less data. Different from the traditional mechanism that obtains personalized demand from historical data; this mechanism introduces the concept of role, establishes mapping relation between roles and requirements, and obtains the personalized needs of users through the role. Because the historical data is the true personalized needs of users and is huge, the personalized needs of users have higher accuracy. The mechanism also has good adaptive ability: the demand set and mapping rules are not static, and keep constantly adjusted with the data quantity increasing.

\section{ACKNOWLEDGMENT}

This work was financially supported by the Foundation projects: Hainan University serving local economic and social development project (project number: HDSF201310), the natural science foundation of Hainan Province(project number: 614232) and the industry-university-research special fund of Hainan Province(project number:CXY20140002).

\section{REFERENCES}

[1] TANG ZHONG JUN,LONG YU LING. "Research on the method for obtaining individual requirements based on Kano model”. Soft Science ,2012, 26(2): 127-128.

[2]ZHANG YUN YANG, YUAN YUAN. "Application of association rules mining in personalized service of university libraries". Journal of University of Tibet (NATURAL SCIENCE EDITION), 2009,24(1) : 85-86.

[3] XIONG WEI, HUANG SI QIN, WU BI FU. "Evaluation of the Stars Hotel website function based on customer demand". Journal of travel, 2009, 9: 61-62.

[4] CUI GUO HUA. "User demand analysis of personalized service in the digital library “. The library circle, 2012, (3) : 40-43.

[5] ZHANG LING YUN, LI NAO, LIU MIN. "The basic concept and the theoretical system of the smart tourism”, Journal of travel, 2012, 27(5) : 6207-6208.

[6] LIU FANG, LIN LA. "Design and research of personalized information service in the tourism electronic commerce system “. Computer knowledge and technology, 2009, 5(22): 6209-6210.

[7] ZHU LING. "Design and implementation of user management system in network”. Computer \& Digital Engineering, 2008, 36 (2) : 162-163 